VOL. 46 (1992) [47-66]

\title{
INVEX OPTIMISATION PROBLEMS
}

\author{
D.T. LuC and C. Malivert
}

In this paper we extend the concept of invexity to set-valued maps and study vector optimisation problems with invex set-valued data. Necessary and sufficient optimality conditions are established in terms of contingent derivatives. Wolfe type dual problems are constructed via two recently developed approaches which guarantee the zero-gap duality property.

\section{INTRODUCTION}

Let $f$ be a convex function on a topological vector space $X$. One of the most useful properties is that, if $f$ is finite and continuous at $x_{0} \in X$, one has

$$
f(x)-f\left(x_{0}\right) \geqslant f^{\prime}\left(x_{0}\right)\left(x-x_{0}\right), \quad \text { for all } x \in X
$$

where $f^{\prime}\left(x_{0}\right)$ stands for the directional derivative at $x_{0}$.

Generalising this property Craven $[3,4,5,6]$, Hanson $[9]$ and others $[2,8,17]$ et cetera have introduced the concept of invexity and studied mathematical programming problems with invex objectives and invex constraints. It is known, for invex problems, traditional necessary optimality criteria are also sufficient. This makes it possible to construct Wolfe type duality which satisfies the zero gap property, between the primal and dual problems. In the mentioned works the theory has been developed for both scalar and multiobjective problems. Even more, in [5] a nonsmooth version was treated, thus justifying the methods of invex functions for a larger class of problems.

The aim of the present work is to extend the study of invexity to set-valued maps and vector optimisation problems with set-valued data. In Section 2 we recall some definitions concerning contigent derivatives of set-valued maps $[1,10,12,15]$. In Section 3 , the concept of invexity is introduced for set-valued maps and several properties of invex maps are provided. The link between invexity and convexity is also given. The rest of the paper deals with vector optimisation problems whose data are invex maps. Optimality conditions are established separately for three cases: unconstrained problems (Section 4), problems with inequality constraints and problems with inequality as

\section{Received 9 July 1991}

The authors would like to express their thanks to Dr. B.D. Craven of Melbourne University for calling their attention to invex optimisation.

Copyright Clearance Centre, Inc. Serial-fee code: 0004-9729/92 \$A2.00+0.00. 
well as equality constraints (Section 5). The last section is devoted to duality theory. By making use of the axiomatic approach of [13] (see also [12]) and the generalised Lagrangian approach of [7] (see also [14]), we obtain several Wolfe type duals for invex problems and verify the exactness of duality.

\section{Contingent Derivatives}

In this section we recall the concept of contingent derivatives of set-valued maps and establish a new result on the derivative of a product of maps which will be needed in the sequel.

Let $X$ and $E$ be two topological vector spaces over $\mathbb{R}$, and $F$ a set-valued map from $X$ to $E$. The contingent derivative of $F$ at a point $\left(x_{0}, y_{0}\right)$ of the graph of $F$ is a set-valued map, denoted by $D F\left(x_{0}, y_{0}\right)$ from $X$ to $E$ whose graph is the contingent cone to the graph of $F$ at the point $\left(x_{0}, y_{0}\right)$; that is: for $u \in X$ one has $v \in D F\left(x_{0}, y_{0}\right)(u)$ if and only if there are a net $\left\{x_{\alpha}, y_{\alpha}\right\}$ from the graph of $F$ converging to $\left(x_{0}, y_{0}\right)$ and a net $\left\{t_{\alpha}\right\}$ of positive numbers converging to $+\infty$ such that

$$
\lim t_{\alpha}\left(x_{\alpha}-x_{0}, y_{\alpha}-y_{0}\right)=(u, v) .
$$

Given a direction $u \in X$, the Dini lower derivative of $F$ at $\left(x_{0}, y_{0}\right)$ in direction $u$ is defined, using the lower Kuratowski-Painlevé limit, by [15]:

$$
D_{\text {low }} F\left(x_{0}, y_{0}\right)(u)=\lim _{\left(t, u^{\prime}\right) \rightarrow\left(0^{+}, u\right)} \frac{F\left(x_{0}+t u^{\prime}\right)-y_{0}}{t} .
$$

It is evident that $D_{\text {low }} F\left(x_{0}, y_{0}\right)(u) \subset D F\left(x_{0}, y_{0}\right)(u)$ for every $u \in X$. Strict inclusion may occur.

For the sake of convenience let us recall also that $F$ is said to be lower semidifferentiable at $\left(x_{0}, y_{0}\right)$ if, for every net $\left\{x_{\alpha}\right\}$ from $X$ converging to $x_{0}$ and every net $\left\{t_{\alpha}\right\}$ of positive numbers such that $\lim t_{\alpha}\left(x_{\alpha}-x_{0}\right)$ exists, there is a subnet $\left\{y_{\alpha_{\beta}}\right\}$, $y_{\alpha_{\beta}} \in F\left(x_{\alpha_{\beta}}\right)$ such that $\lim t_{\alpha_{\beta}}\left(y_{\alpha_{\beta}}-y_{0}\right)$ exists. It was noticed in [11] that if the lower Dini derivative of $F$ at $\left(x_{0}, y_{0}\right)$ is nonempty in every direction of the contingent cone to the $\operatorname{set} \operatorname{dom} F$ at $x_{0}$, denoted by $T\left(\operatorname{dom} F, x_{0}\right)$, then $F$ is lower semidifferentiable. The converse is, in general, not true. We refer the reader to [1] and [11] for more information on contingent derivatives and set-valued maps.

Now, suppose that $G$ is a set-valued map from $X$ to another topological vector space $E_{1}$. The couple $(F, G)$ is a set-valued map from $X$ to $E \times E_{1}$ defined by $(F, G)(x)=(F(x), G(x))$. The following result compares the contingent derivatives and the Dini lower derivatives of $F$ and $G$ with that of the couple $(F, G)$. 
Proposition 2.1. Let $\left(x_{0}, y_{0}\right) \in \operatorname{graph} F,\left(x_{0}, z_{0}\right) \in \operatorname{graph} G$. Then for every $u \in X$ one has

(i) $D_{\text {low }}(F, G)\left(x_{0}, y_{0}, z_{0}\right)(u)=\left(D_{\text {low }} F\left(x_{0}, y_{0}\right), D_{\text {low }} G\left(x_{0}, z_{0}\right)\right)(u)$,

(ii) $\left(D_{\text {low }} F\left(x_{0}, y_{0}\right), D G\left(x_{0}, z_{0}\right)\right)(u) \cup \quad\left(D F\left(x_{0}, y_{0}\right), D_{\text {low }} G\left(x_{0}, z_{0}\right)\right)(u)$ $\subset D(F, G)\left(x_{0}, y_{0}, z_{0}\right)(u) \subset\left(D F\left(x_{0}, y_{0}\right), D G\left(x_{0}, z_{0}\right)\right)(u)$.

PROOF: The first assertion and the second inclusion of the second assertion are evident. Let us prove the first inclusion of the second assertion. Take any $(v, w) \in$ $\left(D_{\text {low }} F\left(x_{0}, y_{0}\right), D G\left(x_{0}, z_{0}\right)\right)(u)$. This means in particular that $w \in D G\left(x_{0}, z_{0}\right)(u)$, that is, there are a net $\left\{\left(x_{\alpha}, z_{\alpha}\right)\right\}$ from graph $G$ converging to $\left(x_{0}, z_{0}\right)$ and a net of positive numbers $\left\{t_{\alpha}\right\}$ converging to $+\infty$ such that $(u, w)=\lim t_{\alpha}\left(x_{\alpha}-x_{0}, z_{\alpha}-z_{0}\right)$. By definition of $D_{\text {low }} F\left(x_{0}, y_{0}\right)$, for two given nets $\left\{x_{\alpha}\right\}$ and $\left\{t_{\alpha}\right\}$ we can find $y_{\alpha} \in$ $F\left(x_{\alpha}\right)$ such that $\lim t_{\alpha}\left(y_{\alpha}-y_{0}\right)=v$. Since $\lim t_{\alpha}=+\infty$, we see that $\lim y_{\alpha}=y_{0}$. Hence $(v, w) \in D(F, G)\left(x_{0}, y_{0}, z_{0}\right)(u)$. The other case where $D_{\text {low }} F$ and $D_{\text {low }} G$ change the roles is proven by the same argument.

Proposition 2.2. Let $C$ be a convex cone in $E$ with a nonempty interior. Then the Dini lower derivative of the map $F+C$ is lower semicontinuous.

Proof: Let $(x, y) \in \operatorname{graph}(F+C),\left(u_{0}, v_{0}\right) \in \operatorname{graph} D_{\text {low }}(F+C)(x, y)$. Let $V$ be any neighbourhood of zero in $E$. We shall prove the existence of a neighbourhood $U$ of zero in $X$ such that

$$
D_{\text {low }}(F+C)(x, y)(u) \cap\left(v_{0}+V\right) \neq \emptyset
$$

for all $u \in u_{0}+U$. To this end, take any $c \in$ int $C \cap V$. By definition of the Dini lower derivatives, for the given neighbourhood of zero $c-$ int $C$, one can find a neighbourhood $U_{1}$ of zero in $X$ and a positive $\varepsilon$ such that

$$
\left((F(x+t u)-y) / t-v_{0}\right) \cap(c-\operatorname{int} C-C) \neq \emptyset,
$$

for all $u \in u_{0}+U_{1}, t \in(0, \varepsilon)$. Let $U$ be any neighbourhood of zero with $U+U \subset U_{1}$. Now in order to complete the proof, it suffices to show that for every $u \in u_{0}+U$,

$$
v_{0}+c \in D_{\mathrm{low}}(F+C)(x, y)(u),
$$

this relation implying (2.1) because $c \in V$. Let $W$ be any neighbourhood of zero in $E$. Since $W-C$ contains - int $C$ and $u+U \subset u_{0}+U+U \subset u_{0}+U_{1}$, it follows from (2.2) that

$$
\left(\left(F\left(x+t u^{\prime}\right)-y\right) / t-v_{0}-c\right) \cap(W-C) \neq \emptyset
$$

for every $u^{\prime} \in u+U, t \in(0, \varepsilon)$. This gives (2.3) and the proof is complete. 


\section{INVEX MAPS}

We preserve the notations of the previous section. Moreover, the space $E$ is supposed to be partially ordered by a convex cone $C \subset E$, suppose to be pointed (that is, $C \cap-C=\{0\})$.

Definition 3.1: Let $\left(x_{0}, y_{0}\right) \in$ graph $F$ and $\eta(x)$ a transformation on $X$ (that is, $\eta(x)$ is a single-valued map from $X$ to $X$ ) which depends, in general, on $x_{0}$ or $\left(x_{0}, y_{0}\right)$ (sometimes we shall write $\eta_{x_{0}}$ or $\eta_{\left(x_{0}, y_{0}\right)}$ to indicate this dependence). Further let $A$ be a set-valued map from $X$ to $E$. We say that $F$ is $A$-invex at $\left(x_{0}, y_{0}\right)$ with respect to $\eta_{\left(x_{0}, y_{0}\right)}$ if for every $x \in X$,

$$
F(x) \subset y_{0}+A\left(\eta_{\left(x_{0}, y_{0}\right)}(x)\right)+C .
$$

If (3.1) holds for every $y_{0} \in F\left(x_{0}\right), F$ is called $A$-invex at $x_{0}$. We shall say simply that $F$ is invex instead of $A$-invex if $A$ coincides with $D F\left(x_{0}, y_{0}\right)$ and that $F$ is $D_{\text {low }}$-invex if $A$ coincides with $D_{\text {low }} F\left(x_{0}, y_{0}\right)$.

The concept of invexity was introduced in several papers of Craven, Hanson and others for Fréchet differentiable functions. In [5] Craven gave a nonsmooth version of invexity as follows. A locally Lipschitz single-valued function $F$, from $\mathbb{R}^{n}$ to $\mathbb{R}^{p}$, is said to be invex at $x_{0}$ with respect to $\eta$, if for every $x \in X$ one has

$$
F(x) \in F\left(x_{0}\right)+\bigcap_{M \in \partial F\left(x_{0}\right)} M(\eta(x))+C,
$$

where $\partial F\left(x_{0}\right)$ is the generalised Jacobian of $F$ at $x_{0}$. Here it is supposed that $X$ and $E$ are finite dimensional spaces so that the generalised Jacobian is a nonempty compact subset of linear operators. We observe that our definition coincides with that of [5] whenever $F$ is Fréchet differentiable at $x_{0}$; however they are different in general: choose for instance $F(x)=-|x| \in \mathbb{R},\left(x_{0}, y_{0}\right)=(0,0)$ and $\eta(x)=x$.

By using properties of contingent derivatives one can easily provide conditions for a sum or a composition of invex maps to be invex. Let us omit this routine work, although we give here a result on the invexity of a product of invex maps which is useful in the study of constrained optimisation problems. It is supposed that the space $E_{1}$ is also partially ordered by a convex cone $K \subset E_{1}$. Then the produce space $E \times E_{1}$ can be ordered by the cone $(C, K)$.

PROposition 3.2. Assume that $F$ is $D_{\text {low }}$-invex at $\left(x_{0}, y_{0}\right) \in$ graph $F$ with respect to $\eta_{x_{0}}$. Then the product map $(F, G)$ is invex (respectively $D_{\text {low }}$-invex) at $\left(x_{0}, y_{0}, z_{0}\right) \in \operatorname{graph}(F, G)$ with respect to $\eta_{x_{0}}$ if $G$ is at $\left(x_{0}, z_{0}\right)$.

Proof: Suppose that $G$ is invex at $\left(x_{0}, z_{0}\right)$ with respect to $\eta_{x_{0}}$. For every $x \in X$, one has

$$
G(x) \in z_{0}+D G\left(x_{0}, z_{0}\right)\left(\eta_{x_{0}}(x)\right)+K
$$


Since $F$ is $D_{\text {low }}$-invex at $\left(x_{0}, y_{0}\right)$, (3.1) holds with $A=D_{\text {low }} F\left(x_{0}, y_{0}\right)$. This and Proposition 2.1 (ii) imply that

$$
(F, G)(x) \subset\left(y_{0}, z_{0}\right)+D(F, G)\left(x_{0}, y_{0}, z_{0}\right)\left(\eta_{x_{0}}(x)\right)+(C, K) .
$$

Hence $(F, G)$ is invex at $\left(x_{0}, y_{0}, z_{0}\right)$ with respect to $\eta_{x_{0}}(x)$. The case where $G$ is $D_{\text {low }}$-invex is proven by the same argument by using Proposition 2.1 (i).

Note that, in the proposition above, the condition that $F$ be $D_{\text {low }}$-invex cannot be weakened. More precisely, it is not difficult to construct two invex maps, the product of which is not invex. For instance, take functions $f$ and $g$ on $\mathbb{R}$ defined by

$$
\begin{array}{lll}
f(x)=0 & \text { and } g(x)=1 /|x| \text { if } x \text { is a nonzero rational number } \\
f(x)=1 /|x| & \text { and } g(x)=0 \quad \text { if } x \text { is an irrational number } \\
f(0)=0, g(0)=0 . & &
\end{array}
$$

Then $f$ and $g$ are invex at 0 with respect to $\eta(x)=x$, while $(f, g)$ is not invex there (the cones, $C$ and $K$ are the set of non-negative numbers).

The next result shows the link between invexity and convexity. Recall that $F$ is $C$-convex if for every $x_{1}, x_{2} \in \operatorname{dom} F, \lambda \in(0,1)$ the following inclusion holds

$$
\lambda F\left(x_{1}\right)+(1-\lambda) F\left(x_{2}\right) \subset F\left(\lambda x_{1}+(1-\lambda) x_{2}\right)+C .
$$

Proposition 3.3. Assume that $F$ is $C$-convex. Then the map $F(x)+C$ is invex at every point $\left(x_{0}, y_{0}\right) \in$ graph $F$ with respect to the transformation $\eta_{x_{0}}(x)=$ $x-x_{0}$. It is $D_{\text {low }}$-invex if in addition $F$ is lower semicontinuous at $x_{0}$.

Conversely, assume that for each $x_{0} \in X$, there exists $y_{0} \in F\left(x_{0}\right)$ such that $F$ is $A$-invex at $\left(x_{0}, y_{0}\right)$ with respect to $\eta_{x_{0}}(x)=x-x_{0}$ and that $A$ is $C$-convex with $A(0) \subset C$. Then $F$ is $C$-convex.

PROOF: To prove the first part of the proposition we have to show that

$$
F(x)+C \subset y_{0}+D(F+C)\left(x_{0}, y_{0}\right)\left(x-x_{0}\right)+C,
$$

for all $x \in X$. It follows from the convexity that

$$
F(x) \subset y_{0}+\left(F\left(x_{0}+\lambda\left(x-x_{0}\right)\right)-y_{0}\right) / \lambda+C
$$

is true for every $x \in S, \lambda \in(0,1)$. The inclusion (3.3) can also be written as

$$
F(x) \subset y_{0}+\left(F\left(x_{0}+\lambda\left(x-x_{0}\right)\right)+C-y_{0}\right) / \lambda
$$


which implies (3.2), since by convexity, for each $z \in X,\left(z-x_{0}, F(x)+C-y_{0}\right)$ belongs to the contingent cone at $\left(x_{0}, y_{0}\right)$ to the set $F+C$. Thus $F(x)+C$ is invex. Now suppose in addition that $F$ is lower semicontinuous at $x_{0}$ and let $w$ be any element of the set $F\left(x_{0}+\lambda\left(x-x_{0}\right)\right)-y_{0}$. For a fixed number $\lambda \in(0,1)$, for any neighbourhood $V$ of $w$ there exists a neighbourhood $U$ of $\lambda\left(x-x_{0}\right)$ such that $F\left(x_{0}+u\right) \cap\left(V+y_{0}\right) \neq \emptyset$ whenever $u \in U$. This and the convexity of $F$ imply

$$
\left(F\left(x_{0}+t u\right)-y_{0}\right) / t \cap(V-C) \neq \emptyset
$$

for every $t \in(0,1)$. Thus, $w \in D_{\text {low }}(F+C)\left(x_{0}, y_{0}\right)\left(\lambda\left(x-x_{0}\right)\right)$, or equivalently, $w / \lambda \in$ $D_{\text {low }}(F+C)\left(x_{0}, y_{0}\right)\left(x-x_{0}\right)$. We have established the inclusion

$$
F\left(x_{0}+\lambda\left(x-x_{0}\right)\right) \subset D_{\text {low }}(F+C)\left(x_{0}, y_{0}\right)\left(x-x_{0}\right)
$$

which together with (3.3) shows that $F(x)+C$ is $D_{\text {low }}$-invex at $\left(x_{0}, y_{0}\right)$ with respect to $\eta(x)=x-x_{0}$.

For the second part, let $x_{1}, x_{2} \in \operatorname{dom} F$ and $\lambda \in(0,1)$. Set $x_{0}=\lambda x_{1}+(1-\lambda) x_{2}$ and suppose that $y_{0} \in F\left(x_{0}\right)$ is the point stated in the proposition. By the invexity,

$$
F\left(x_{i}\right) \subset y_{0}+A\left(x_{i}-x_{0}\right)+C, \quad i=1,2 .
$$

Using this and the properties of $A$ we can write the following inclusions:

$$
\begin{aligned}
\lambda F\left(x_{1}\right)+(1-\lambda) F\left(x_{2}\right) & \subset y_{0}+\lambda A\left(x_{1}-x_{0}\right)+(1-\lambda) A\left(x_{2}-x_{0}\right)+C \\
& \subset F\left(x_{0}\right)+C .
\end{aligned}
$$

Hence $F$ is $C$-convex and the proof is complete.

\section{OPTIMALITY CONDITIONS Without CONSTRAints}

In this section we assume that $C$ is pointed with a nonempty interior int $C$. We consider the following problem

$$
\begin{aligned}
& \min F(x) \\
& x \in X
\end{aligned}
$$

We recall that the set of weak efficient values of $(P)$ is the set

$$
W \operatorname{Min} F(X):=\{a \in F(X): F(X) \cap(a-\text { int } C)=\emptyset\}
$$

and a point $\left(x_{0}, y_{0}\right) \in \operatorname{graph} F$ is called a weak minimiser of $(\mathrm{P})$ if $y_{0}$ is a weak efficient value of $(\mathrm{P})$.

In the sequel we shall provide optimality criteria for problem (P) under invexity assumptions. 
Theorem 4.1. Let $\left(x_{0}, y_{0}\right) \in$ graph $F$. Assume that $F$ is $A$-invex at $\left(x_{0}, y_{0}\right)$ where $A$ stands for $D F\left(x_{0}, y_{0}\right)$ or $D_{\text {low }} F\left(x_{0}, y_{0}\right)$. Then $\left(x_{0}, y_{0}\right)$ is a weak minimiser of $(P)$ if and only if, for all $u \in X$,

$$
A(u) \cap \text {-int } C=\emptyset .
$$

Proof: The necessary condition was established in $[11,12]$ for $D F\left(x_{0}, y_{0}\right)$ even without the invexity assumption. Since $A(u) \subset D F\left(x_{0}, y_{0}\right)(u)$ for every $u \in X$, the relation (4.1) follows. For the sufficient condition, supposing to the contrary that $\left(x_{0}, y_{0}\right)$ is not a weak minimiser, one can find a point $(x, y) \in$ graph $F$ such that $y \in y_{0}-$ int $C$. By the invexity,

$$
F(x) \subset y_{0}+A\left(\eta_{\left(x_{0}, y_{0}\right)}(x)\right)+C,
$$

where $\eta_{\left(x_{0}, y_{0}\right)}$ is a transformation with respect to which $F$ is $A$-invex. Hence, there exist some vectors $v \in A\left(\eta_{\left(x_{0}, y_{0}\right)}(x)\right), c \in C$ such that

$$
y=y_{0}+v+c .
$$

This implies $v=y-y_{0}-c \in-\operatorname{int} C-C \subset-\operatorname{int} C$ which contradicts (4.1).

REMARK 4.2. It is clear that $\left(x_{0}, y_{0}\right) \in$ graph $F$ is a weak minimiser of (P) if and only if it is a weak minimiser of the problem

$$
\begin{aligned}
& \min F(x)+C . \\
& x \in X
\end{aligned}
$$

Hence, Theorem 4.1 remains valid if $F(x)$ is replaced by $F(x)+C$.

We recall that $\left(x_{0}, y_{0}\right) \in$ graph $F$ is said to be a local weak minimiser of $(\mathrm{P})$ if it is a weak minimiser of the problem

$$
\begin{aligned}
& \min F(x) \\
& x \in X \cap U
\end{aligned}
$$

where $U$ is some neighbourhood of $x_{0}$ in $X$.

Corollary 4.3. Let $\left(x_{0}, y_{0}\right) \in$ graph $F$ be a local weak minimiser of $(P)$. It is also a weak minimiser of the problem if the map $F$ is $A$-invex at $\left(x_{0}, y_{0}\right)$ where $A$ stands for $D F\left(x_{0}, y_{0}\right)$ or $D_{\text {low }} F\left(x_{0}, y_{0}\right)$.

Proof: In fact, as it was shown in [12], formula (4.1) is a necessary condition for local weak minimisers. Hence in the case where $F$ is $A$-invex, Theorem 4.1 assures us that the point is a global weak minimiser of the problem. 


\section{OPTIMALITY CONDITIONS WITH EXPLICIT CONSTRAINTS}

Let us consider the constrained problem:

$$
\begin{aligned}
& \min F(x) \\
& G(x) \cap-K \neq \emptyset \\
& 0 \in P(x)
\end{aligned}
$$

where $F$ and $G$ are, as before, set-valued maps from $X$ to $E$ and $E_{1}, P$ is a new set-valued map from $X$ to $E_{2}$ and $K$ a convex cone. The conditions (5.1) and (5.2) generalise inequality and equality constraints in mathematical programming.

We recall that a point $\left(x_{0}, y_{0}, z_{0}\right) \in X \times E \times E_{1}$ is said to be a feasible triple of (CP) (in this case $x_{0}$ is called a feasible solution) if $y_{0} \in F\left(x_{0}\right), z_{0} \in G\left(x_{0}\right) \cap-K$ and $0 \in P\left(x_{0}\right)$. A feasible triple $\left(x_{0}, y_{0}, z_{0}\right)$ is called a weak minimiser of (CP) if,

$$
y_{0} \in W \operatorname{Min}\{F(x): x \text { is a feasible solution of }(\mathrm{CP})\}
$$

Observe that the space $E \times E_{1} \times E_{2}$ may be considered as a partially ordered space whose order is defined by the cone $(C, K,\{0\})$. Let $A$ be a set-valued map from $X$ to $E \times E_{1} \times E_{2}$ and $L\left(E_{1}, E\right)$ the space of linear operators from $E_{1}$ to $E$, while $L_{+}\left(E_{1}, E\right)$ consists of those which carry $K$ into $C$.

ThEOREM 5.1. Suppose that the following conditions hold at a feasible triple $\left(x_{0}, y_{0}, z_{0}\right)$ of $(C P)$ :

(i) The map $(F, G, P)$ is $A$-invex at $\left(x_{0}, y_{0}, z_{0}, 0\right)$ with respect to $\eta_{\left(x_{0}, y_{0}, z_{0}\right)}$.

(ii) For every $u \in \operatorname{dom} A$ and every $(v, w, r) \in A(u)$ there exist linear operators $\ell \in L_{+}\left(E_{1}, E\right), h \in L\left(E_{2}, E\right)$ such that

$$
v+\ell\left(w+z_{0}\right)+h(r) \notin-\text { int } C .
$$

Then $\left(x_{0}, y_{0}, z_{0}\right)$ is a weak minimiser of $(C P)$.

Proof: Supposing to the contrary that $\left(x_{0}, y_{0}, z_{0}\right)$ is not a weak minimiser of (CP) we can find a feasible triple $(x, y, z)$ of (CP) such that

$$
y \in y_{0}-\text { int } C \text {. }
$$

By the invexity we have, denoting simply $\eta$ instead of $\eta_{\left(x_{0}, y_{0}, z_{0}\right)}$,

$$
(F, G, P)(x) \subset\left(y_{0}, z_{0}, 0\right)+A(\eta(x))+(C, K,\{0\}) .
$$

In particular,

$$
(y, z, 0) \in\left(y_{0}, z_{0}, 0\right)+A(\eta(x))+(C, K,\{0\})
$$


which means that there exist vectors $(v, w, r) \in A(\eta(x))$ and $(c, k, 0) \in(C, K,\{0\})$ such that $(y, z, 0)=\left(y_{0}, z_{0}, 0\right)+(v, w, r)+(c, k, 0)$. This implies the equality $v=$ $y-y_{0}-c, w+z_{0}=z-k$ and $r=0$.

These latter relations together with (5.4) show that

$$
v+\ell\left(w+z_{0}\right)+h(r) \in-\text { int } C,
$$

which contradicts (5.3).

REMARK 5.2. It is useful to observe that a feasible triple $(x, y, z)$ of $(\mathrm{CP})$ is a weak minimiser if and only if it is a weak minimiser of the problem obtained from (CP) by substituting $F(x)+C$ and $G(x)+K$ in the place of $F(x)$ and $G(x)$ respectively. So, the result of the previous theorem is still valid if $F(x)$ and $G(x)$ are replaced by $F(x)+C$ and $G(x)+K$.

REMARK 5.3. The result of Theorem 5.1 does not change if the second condition is replaced by the following one:

(iii) For every $u \in \operatorname{dom} A$ and every $(v, w, r) \in A(u)$ there exists a vector $(\xi, \zeta, \nu) \in(C, K,\{0\})^{\prime}$ with $\xi \neq 0$ such that

$$
\xi(v)+\zeta\left(w+z_{0}\right)+\nu(r) \geqslant 0
$$

where $(C, K,\{0\})^{\prime}$ is the nonnegative dual cone of $(C, K,\{0\})$. This fact can be derived from the following lemma.

Lemma 5.4. Suppose that (5.5) is true. Then it is possible to construct linear operators $\ell \in L_{+}\left(E_{1}, E\right), h \in L\left(E_{2}, E\right)$ which satisfy (5.3).

Proof: Pick any $e \in$ int $C$ with $\xi(e)=1$. (Such a vector exists because $\xi \in C^{\prime}$ and $\xi \neq 0)$. For every $(w, r) \in E_{1} \times E_{2}$ define $\ell(w)=\zeta(w)$.e and $h(r)-\nu(r)$.e. It is obvious that $\ell$ and $h$ satisfy our requirements.

In the rest of this section we shall provide necessary conditions for (CP). No invexity assumptions are needed from now on. Let us consider first, the case where the equality constraint (5.2) is absent, that is, we are dealing with the problem

$$
\min F(x)
$$

$$
G(x) \cap-K \neq \emptyset,
$$

where $K$ is assumed to have a nonempty relative interior.

Theorem 5.5. Let $\left(x_{0}, y_{0}, z_{0}\right)$ be a (local) weak minimiser of $\left(C P_{1}\right)$. Then for every $u \in \operatorname{dom} D(F, G)\left(x_{0}, y_{0}, z_{0}\right)$, every $(v, w) \in D(F, G)\left(x_{0}, y_{0}, z_{0}\right)(u)$, there exists a nonzero vector $(\xi, \zeta) \in(C, K)^{\prime}$ such that

$$
\xi(v)+\zeta\left(w+z_{0}\right) \geqslant 0 .
$$


Proof: Suppose to the contrary that there exist $u$ and $(u, w)$ as above, such that for all nonzero $(\xi, \zeta) \in(C, K)^{\prime}$ one has

$$
\xi(v)+\zeta\left(w+z_{0}\right)<0
$$

This, in particular, implies that

$$
v \in-\text { int } C \text { and } w+z_{0} \in-K
$$

If int $K=\emptyset$, since ri $K \neq \emptyset$, there exists a nonzero vector $\zeta \in K^{\prime}$ such that $\zeta(k)=0$ for every $k \in K$. By taking this $\zeta$ and $\xi \equiv 0$ in (5.7) we get the contradiction $\xi(v)+\zeta\left(w+z_{0}\right)=0$. Hence one may assume that int $K \neq \emptyset$. In this case (5.7) implies that

$$
w+z_{0} \in-\text { int } K
$$

By definition of contingent derivatives, for $u$ and $(v, w)$ as above, one can find a net $\left\{\left(x_{\alpha}, y_{\alpha}, z_{\alpha}\right)\right\}$ from graph $(F, G)$ converging to $\left(x_{0}, y_{0}, z_{0}\right)$ and a net of positive numbers $t_{\alpha}$ with $\lim t_{\alpha}=\infty$, such that $\lim t_{\alpha}\left(x_{\alpha}-x_{0}, y_{\alpha}-y_{0}, z_{\alpha}-z_{0}\right)=(u, v, w)$. The relations (5.8) and (5.9) show that for $\alpha$ large enough, $t_{\alpha}\left(y_{\alpha}-y_{0}\right) \in-$ int $C$ and $t_{\alpha}\left(z_{\alpha}-z_{0}\right)+z_{0} \in-$ int $K$. In other words, $y_{\alpha} \in y_{0}-$ int $C$ and $z_{\alpha} \in z_{0}-z_{0} / t_{\alpha}-$ int $K$. Since $\lim \left(z_{0} / t_{\alpha}\right)=0$ and $z_{0} \in-K$, the latter relation shows that $z_{\alpha} \in-K$ for $\alpha$ large enough. Thus, for these $\alpha,\left(x_{\alpha}, y_{\alpha}, z_{\alpha}\right)$ are feasible triples with $y_{\alpha} \in y_{0}-$ int $C$. This contradicts the fact that $\left(x_{0}, y_{0}, z_{0}\right)$ is a local weak minimiser of $\left(\mathrm{CP}_{1}\right)$.

The theorem above is an improvement of Corollary 3.3 of [16] for the reason that $F$ and $G$ need not be lower semidifferentiable. Observe also that under the conditions of the mentioned corollary, the last inclusion in (ii) of Proposition 2.1 becomes an equality and in fact (5.6) is true for every $v \in D F\left(x_{0}, y_{0}\right)(u), w \in D G\left(x_{0}, z_{0}\right)(u)$.

THEOREM 5.6. Assume that $\left(x_{0}, y_{0}, z_{0}\right)$ is a local weak minimiser of $\left(C P_{1}\right)$ and int $K$ is nonempty. For any vector $u \in \operatorname{dom} D(F, G)\left(x_{0}, y_{0}, z_{0}\right)$, if the set $D(F, G)\left(x_{0}, y_{0}, z_{0}\right)(u)+(C, K)$ is convex, then there exists a nonzero vector $(\xi, \zeta) \in$ $(C, K)^{\prime}$ such that for all $(v, w) \in D(F, G)\left(x_{0}, y_{0}, z_{0}\right)(u)$ one has

$$
\xi(v)+\zeta\left(w+z_{0}\right) \geqslant 0
$$

Moreover, if the set $D(F, G)\left(x_{0}, y_{0}, z_{0}\right)(X)+(C, K)$ is convex, then there exists a nonzero vector $(\xi, \zeta) \in(C, K)^{\prime}$ such that $(5.10)$ is true for all $u \in X$ and $\zeta\left(z_{0}\right)=0$.

The vector $\xi$ can be assured nonzero under an additional hypothesis called constraint qualification:

$$
D(F, G)\left(x_{0}, y_{0}, z_{0}\right)(X) \cap(- \text { int } K) \neq \emptyset
$$


Proof: It is clear that at a local weak minimiser $\left(x_{0}, y_{0}, z_{0}\right)$, one has

$$
D(F, G)\left(x_{0}, y_{0}, z_{0}\right)(u) \cap\left(-\operatorname{int} C, z_{0}-\text { int } K\right)=\emptyset, \text { for all } u \in X .
$$

Under the convexity assumption, one can separate these sets by a nonzero vector $(\xi, \zeta) \in(C, K)^{\prime}$. The inequality $(5.10)$ is then immediate. In the case where the set $D(F, G)\left(x_{0}, y_{0}, z_{0}\right)(X)+(C, K)$ is convex, by taking $v=0, w=0$ (which corresponds to $u=0$ ) we obtain from (5.10) that $\zeta\left(z_{0}\right) \geqslant 0$. Remembering that $z_{0} \in-K$ and $\zeta \in K^{\prime}$, we conclude $\zeta\left(z_{0}\right)=0$. Finally, under the constraint qualification, $\xi$ cannot be zero, otherwise a nonzero vector $\zeta$ should separate the projection of $D(F, G)\left(x_{0}, y_{0}, z_{0}\right)(X)$ on $E_{1}$ and the set - int $K$, and the intersection in (5.11) should be empty.

Let us now study the case of Problem (CP) where $P$ is a single-valued map, the Fréchet derivative of which exists and is surjective. We denote this derivative by $P^{\prime}$. The spaces $X$ and $E_{2}$ are now assumed to be Banach spaces.

THEOREM 5.7. Let $\left(x_{0}, y_{0}, z_{0}\right)$ be a local weak minimiser of $(C P)$. Then for every $u \in X$ with $P^{\prime}\left(x_{0}\right)(u)=0$ and for every $(v, w) \in D_{\text {low }}(F, G)\left(x_{0}, y_{0}, z_{0}\right)$, there exists a nonzero vector $(\xi, \zeta) \in(C, K)^{\prime}$ such that (5.6) holds.

Proof: Suppose to the contrary that the statement is not true. As in the proof of Theorem 5.5, for some $u \in X,(v, w) \in D_{\text {low }}(F, G)\left(x_{0}, y_{0}, z_{0}\right)(u)$ one has $P^{\prime}\left(x_{0}\right)(u)=$ $0, v \in-$ int $C$ and

$$
w+z_{0} \in-\text { int } K
$$

In view of the Lyusternik theorem, there are a net $\left\{x_{\alpha}\right\}$ converging to $x_{0}$, a net of positive numbers $\left\{t_{\alpha}\right\}$ converging to $+\infty$ such that $P\left(x_{\alpha}\right)=0$ and $\lim t_{\alpha}\left(x_{\alpha}-x_{0}\right)=u$. Then by definition of lower Dini derivatives, there exists a net $\left\{\left(y_{\alpha}, z_{\alpha}\right)\right\},\left(y_{\alpha} \in F\left(x_{\alpha}\right)\right.$, $\left.z_{\alpha} \in G\left(x_{\alpha}\right)\right)$, converging to $\left(y_{0}, z_{0}\right)$ such that $\lim t_{\alpha}\left(y_{\alpha}-y_{0}, z_{\alpha}-z_{0}\right)=(v, w)$. Hence, for $\alpha$ sufficiently large, (5.12) implies that

$$
t_{\alpha}\left(y_{\alpha}-y_{0}, z_{\alpha}-z_{0}\right) \in\left(-\operatorname{int} C,-z_{0}-\operatorname{int} K\right) .
$$

In other words, $y_{\alpha} \in y_{0}-$ int $C$ and $z_{\alpha} \in z_{0}-z_{0} / t_{\alpha}-$ int $K$. When $\alpha$ is large, $t_{\alpha}$ is large too, and the latter inclusion with the fact $z_{0} \in-K$ show that $z_{\alpha} \in-K$. In this way, we have found a feasible triple $\left(x_{\alpha}, y_{\alpha}, z_{\alpha}\right)$ of $(\mathrm{CP})$ for which

$$
y_{\alpha} \in y_{0}-\text { int } C \text {. }
$$

This means that $\left(x_{0}, y_{0}, z_{0}\right)$ cannot be a local weak minimiser of (CP). The contradiction completes the proof. 
TheOREM 5.8. Assume that $\left(x_{0}, y_{0}, z_{0}\right)$ is a local weak minimiser of $(C P)$ and int $K$ is nonempty. For a vector $u \in \operatorname{dom} D_{\text {low }}(F, G)\left(x_{0}, y_{0}, z_{0}\right)$ with $P^{\prime}\left(x_{0}\right)(u)=0$, if the set $D_{\text {low }}(F, G)\left(x_{0}, y_{0}, z_{0}\right)(u)+(C, K)$ is convex, then there exists a nonzero vector $(\xi, \zeta) \in(C, K)^{\prime}$ such that (5.10) holds for every $(v, w) \in D_{\text {low }}(F, G)\left(x_{0}, y_{0}, z_{0}\right)(u)$. Moreover, if the set $D_{\text {low }}(F+C, G+K)\left(x_{0}, y_{0}, z_{0}\right)(X)+(C, K)$ is convex, there exists a nonzero vector $(\xi, \zeta, \nu) \in(C, K,\{0\})^{\prime}$ such that $\zeta\left(z_{0}\right)=0$ and

$$
\xi(v)+\zeta(w)+\nu(r) \geqslant 0
$$

for every $(v, w, r) \in D_{\text {low }}(F+C, G+K, P)\left(x_{0}, y_{0}, z_{0}, 0\right)(X)$.

The vector $\xi$ is nonzero under the following hypothesis:

$$
D_{\text {low }}(F+C, G+K, P)\left(x_{0}, y_{0}, z_{0}, 0\right)(X) \cap(E,- \text { int } K,\{0\}) \neq \emptyset \text {. }
$$

Proof: Observe first that for every $u \in X$, one has

$$
D_{\text {low }}(F, G, P)\left(x_{0}, y_{0}, z_{0}, 0\right)(u) \cap\left(- \text { int } C, z_{0}-\text { int } K,\{0\}\right)=\emptyset \text {. }
$$

By Proposition 2.1 and the fact $D_{\text {low }} P\left(x_{0}, 0\right)(u)=P^{\prime}\left(x_{0}\right)(u)$,

$$
D_{\text {low }}(F, G, P)\left(x_{0}, y_{0}, z_{0}, 0\right)(u)=\left(D_{\text {low }}(F, G)\left(x_{0}, y_{0}, z_{0}\right)(u), P^{\prime}\left(x_{0}\right)(u)\right) .
$$

Hence, for $u \in X$ with $P^{\prime}\left(x_{0}\right)(u)=0$, we have

$$
\left(D_{\text {low }}(F, G)\left(x_{0}, y_{0}, z_{0}\right)(u)+(C, K)\right) \cap\left(-\operatorname{int} C, z_{0}-\text { int } K\right)=\emptyset \text {. }
$$

It suffices now to apply the Hahn-Banach separation theorem to get the assertion of the first part.

For the second part of the theorem we show, at first, that the set

$$
Q=D_{\text {low }}(F+C, G+K, P)\left(x_{0}, y_{0}, z_{0}, 0\right)(X)+\left(\text { int } C, z_{0}+\text { int } K,\{0\}\right)
$$

is open in the space $E \times E_{1} \times E_{2}$. Consider $q_{0}=\left(v_{0}+c_{0}, z_{0}+w_{0}+k_{0}, P^{\prime}\left(x_{0}\right)\left(u_{0}\right)\right) \in$ $Q,\left(v_{0}, w_{0}\right) \in D_{\text {low }}(F+C, G+K)\left(x_{0}, y_{0}, z_{0}\right)\left(u_{0}\right), c_{0} \in \operatorname{int} C, k_{0} \in \operatorname{int} K, u_{0} \in X$. In virtue of Proposition 2.2, there exists a neighbourhood $U$ of $u_{0}$ such that

$$
D_{\text {low }}(F+C, G+K)\left(x_{0}, y_{0}, z_{0}\right)(u) \cap\left(v_{0}+\frac{c_{0}}{2}-\text { int } C, w_{0}+\frac{k_{0}}{2}-\text { int } K\right) \neq \emptyset
$$

for every $u \in U$. By the assumptions of the theorem, the map $P$ is open, that is $P^{\prime}\left(x_{0}\right)(U)$ contains an open set, say $R$. Then (5.14) shows that the open set $\left(v_{0}+c_{0} / 2+\right.$ int $C, z_{0}+w_{0}+k_{0} / 2+$ int $\left.K, R\right)$ contains $q_{0}$ and is contained in $Q$. 
Furthermore, $Q$ does not contain zero because $\left(x_{0}, y_{0}, z_{0}\right)$ is a local weak minimiser. The closure of $Q$ is the same as the closure of the convex set

$$
\left(D_{\text {low }}(F+C, G+K)\left(x_{0}, y_{0}, z_{0}\right), P^{\prime}\left(x_{0}\right)\right)(X)+(C, K,\{0\}),
$$

hence is convex. By the separation theorem there is a nonzero vector $(\xi, \zeta, \nu) \in$ $\left(E \times E_{1} \times E_{2}\right)^{\prime}$ such that $(\xi, \zeta, \nu)(q) \geqslant 0$, for every $q \in Q$. This implies that $(\xi, \zeta, \nu) \in(C, K,\{0\})^{\prime}$ and $\xi(v)+\zeta\left(w+z_{0}\right)+\nu(r) \geqslant 0$ for every $(v, w, r) \in$ $D_{\text {low }}(F+C, G+K, P)\left(x_{0}, y_{0}, z_{0}, 0\right)(X)$. In particular, $\zeta\left(z_{0}\right) \geqslant 0$. This, and the fact that $z_{0} \in-K$ imply $\zeta\left(z_{0}\right)=0$. Finally, it is evident that $\xi \neq 0$ under the hypothesis (5.13).

\section{Duality}

The purpose of this part is to construct dual problems of Wolfe type for a given optimisation problem, whose objective and constraints are invex. The construction of duals is based on the approach called axiomatic duality proposed in $[11,13]$ and the approach of $[7,14]$. Let us consider the problem

$$
\begin{aligned}
& \min F(x) \\
& x \in X_{0}
\end{aligned}
$$

where $X_{0}$ is a subset of $X$.

By a dual of $\left(\mathrm{P}_{0}\right)$, we mean any maximisation problem

$$
\begin{aligned}
& \max H(s) \\
& s \in S
\end{aligned}
$$

where $S$ is a nonempty set, $H$ is a set-valued map from $S$ to $E$, which satisfies the following weak duality relation

$$
H(s) \cap(F(x)+C \backslash\{0\})=\emptyset
$$

for every $s \in S, x \in X_{0}$.

Being a dual of $\left(\mathrm{P}_{0}\right)$, Problem (D) is called an exact dual if

$$
H(S) \cap F\left(X_{0}\right) \neq \emptyset \text {. }
$$

It is evident that (D) is an exact dual of $\left(P_{0}\right)$ if and only if it is a dual and the two problems have common values. 
REMARK 6.1. Let $H_{0}$ be a submap of $H$, that is, graph $H_{0} \subset$ graph $H$. Then the subproblem,

$$
\max _{s \in S} H_{0}(s)
$$

is also a dual of $\left(P_{0}\right)$ whenever (D) is. However it is not necessarily exact if (D) is.

The very purpose of any duality in optimisation is to construct $H$ and $S$ such that (D) is an exact dual of $\left(P_{0}\right)$. Let us first apply the method of $[11,13]$. For the sake of convenience we consider the problem

$$
\begin{aligned}
& \min F(x) \\
& x \in X_{0}, G(x) \cap-K \neq \emptyset .
\end{aligned}
$$

Many of the results to be established are true without requiring int $K \neq \emptyset$, so in fact problems with the presence of equality constraint (5.2) can be written in the form of $\left(\mathrm{CP}_{0}\right)$ by considering the cone $(K,\{0\})$ instead of $K$ and therefore not much loss of generality occurs when we restrict the study to $\left(\mathrm{CP}_{0}\right)$ alone.

Let $E_{0}$ be a topological vector space which is partially ordered by a convex cone $M$, not identical with a linear subspace of $E_{0}$. Denote by $\sum$ the set of single-valued maps $s$ from $E \times E_{1}$ to $E_{0}$ which possess the property that $s(a, b)$ is increasing in the first variable when the second one is zero, that is, for any $a, a^{\prime} \in E, a^{\prime}>a$ implies $s\left(a^{\prime}, 0\right)>s(a, 0)$.

As it was proven in $[11,13]$, taking any $S \subset \Sigma$ one obtains a dual for $\left(\mathrm{CP}_{0}\right)$ :

$$
\begin{aligned}
& \max H(s) \\
& s \in S
\end{aligned}
$$

where $H(s)=\left\{d \in E: s(d, 0) \in \operatorname{Min} s\left(Q\left(X_{0}\right)\right)\right\}$ and $Q\left(X_{0}\right)=\{(F(x)+C, G(x)+K)$ : $\left.x \in X_{0}\right\}$.

The two following cases are of most interest:

(a) $E_{0}=E, M=C$;

(b) $E_{0}=\mathbb{R}, M=\mathbb{R}_{+}$.

We consider first the case (a).

For each vector $(x, y, z) \in \operatorname{graph}(F, G)$, let $A_{(x, y, z)}$ be a homogeneous submap of $D(F, G)(x, y, z)$, and let $\eta_{(x, y, z)}$ be a transformation on $X$. As before, $L_{+}\left(E_{1}, E\right)$ is the set of non-decreasing continuous linear operators $h$ from $E_{1}$ to $E$. We also denote by $L_{x}(X, E)$ the set of continuous linear operators $h$ from $X$ to $E$ with the property $h\left(T\left(X_{0}, x\right)\right) \subset C$ where $T\left(X_{0}, x\right)$ is the contingent cone to $X_{0}$ at $x$. (It 
is supposed, of course, in this case that $\left.x \in X_{0}\right)$; further, consider two multivalued mappings $L_{1}$ and $L_{2}$ from $L_{+}\left(E_{1}, E\right)$ into graph $(F, G)$, defined by:

$$
\begin{aligned}
L_{1}(\ell)= & \{(x, y, z) \in \operatorname{graph}(F, G) \text { such that } \\
& \left.v+\ell w \subset(-C \backslash\{0\})^{c} \text { for all }(v, w) \in A_{(x, y, z)}\left(\eta_{(x, y, z)}\left(X_{0}\right)\right)\right\} \\
L_{2}(\ell)= & \left\{(x, y, z) \in \operatorname{graph}(F, G) \text { such that there exists } h \in L_{x}(X, E)\right. \text { with } \\
& v+\ell w-h\left(\eta_{(x, y, z)}\left(x^{\prime}\right)\right) \subset(-C \backslash\{0\})^{c} \\
& \text { for all } \left.(v, w) \in A_{(x, y, z)}\left(\eta_{(x, y, z)}\left(X_{0}\right)\right), x^{\prime} \in X_{0}\right\}
\end{aligned}
$$

With $L_{1}$ and $L_{2}$ in hand we are able to define two subsets $S_{1}$ and $S_{2}$ of $\Sigma$ as follows: $S_{i}$ consists of single-valued maps $s$ from $E \times E_{1}$ to $E$ of the form

$$
s(a, b)=a+\ell(b), \text { for all }(a, b) \in E \times E_{1},
$$

where $\ell$ is such that $L_{i}(\ell) \neq \emptyset$.

That $S_{1}$ and $S_{2}$ are subsets of $\Sigma$ is obvious because $s(a, 0)=a$, hence it is increasing in $a$. Consequently, the problem (DS) with $S$ being one of these subsets will provide a dual for $\left(\mathrm{CP}_{0}\right)$. Denote these duals by $\left(\mathrm{DS}_{1}\right)$ and $\left(\mathrm{DS}_{2}\right)$. In the sequel we shall show that $\left(D S_{1}\right)$ and $\left(D S_{2}\right)$ provide subproblems written in the familar form of Wolfe type duality. By saying $(F, G)$ is $A$-invex on $X_{0}$ we mean that it is $A_{(x, y, z)}$-invex with respect to $\eta_{(x, y, z)}$ at every $(x, y, z) \in \operatorname{graph}(F, G), x \in X_{0}$.

Theorem 6.2. Assume that $(F, G)$ is $A$-invex on $X_{0}$. Then the dual $\left(D S_{1}\right)$ can be written in the form

$$
\max H_{1}(s)
$$

$$
s \in S_{1}
$$

where

$$
H_{1}(s)=\left\{y+\ell z:(x, y, z) \in L_{1}(\ell), x \in X_{0}\right\}
$$

and $\ell$ is the map which determines $s$.

Moreover, if, in addition, Problem $\left(C_{0}\right)$ possesses a feasible triple $(x, y, z) \in$ $L_{1}(\ell)$ with $\ell(z)=0$, then $\left(D S_{1}\right)$ is an exact dual of $\left(C P_{0}\right)$.

Proof: By definition of $H(s)$ it can be seen that

$$
H(s)=\operatorname{Min} \bigcup_{x^{\prime} \in X_{0}}\left\{F\left(x^{\prime}\right)+\ell G\left(x^{\prime}\right)\right\}
$$


where $\ell$ is the linear operator which determines $s \in S_{1}$. We prove that $H_{1}(s) \subset H(s)$. In fact, if not, there would exist a triple $(x, y, z) \in L_{1}(\ell)$ and $\left(x^{\prime}, y^{\prime}, z^{\prime}\right) \in \operatorname{graph}(F, G)$ with $x^{\prime} \in X_{0}$ such that

$$
y+\ell z-\left(y^{\prime}+\ell z^{\prime}\right) \in C \backslash\{0\}
$$

By the invexity assumption,

$$
\left(y^{\prime}, z^{\prime}\right) \in(y, z)+A_{(x, y, z)}\left(\eta_{(x, y, z)}\left(x^{\prime}\right)\right)+(C, K)
$$

Taking into account the fact that $\ell \in L_{+}\left(E_{1}, E\right)$, the latter relation implies

$$
y^{\prime}+\ell z^{\prime}-(y+\ell z) \in(v+\ell w)+C
$$

for some $(v, w) \in A_{(x, y, z)}\left(\eta_{(x, y, z)}\left(x^{\prime}\right)\right)$. This and the fact that $(x, y, z) \in L_{1}(\ell)$ yield

$$
y+\ell z-\left(y^{\prime}+\ell z^{\prime}\right) \in-(-C \backslash\{0\})^{c}-C \subset(C \backslash\{0\})^{c}
$$

which contradicts (6.3). Finally, for a feasible triple $(x, y, z) \in L_{1}(\ell)$ with $\ell z=0$ one has $y \in F(x) \cap H_{1}(s)$, where $s$ determined by $\ell$. Hence $\left(\mathrm{DS}_{1}\right)$ is an exact dual of $\left(\mathrm{CP}_{0}\right)$.

Theorem 6.3. Assume that $(F, G)$ is $A$-invex on $X_{0}$ and $\eta_{(x, y, z)}\left(X_{0}\right) \subset$ $T\left(X_{0}, x\right)$ for every $x \in X_{0}$. Then the dual $\left(D S_{2}\right)$ can be written in the form

$$
\max H_{2}(s)
$$

$$
s \in S_{2}
$$

where

$$
H_{2}(s)=\left\{y+\ell z:(x, y, z) \in L_{2}(\ell), x \in X_{0}\right\}
$$

and $\ell$ is the map which determines $s$.

Moreover, $\left(\mathrm{DS}_{2}\right)$ is an exact dual of $\left(\mathrm{CP}_{0}\right)$ provided Problem $\left(C P_{0}\right)$ possesses a feasible triple $(x, y, z) \in L_{2}(\ell)$ with $\ell(z)=0$.

Proof: As in the proof of Theorem 6.2, we show that $H_{2}(s) \subset H(s)$. By invexity, for every $\left(x^{\prime}, y^{\prime}, z^{\prime}\right) \in \operatorname{graph}(F, G), x^{\prime} \in X_{0}$ and every feasible solution $\ell$ of $\left(\mathrm{DS}_{2}\right)$, one has

$$
\left(y^{\prime}, z^{\prime}\right) \in(y, z)+A_{(x, y, z)}\left(\eta(x, y, z)\left(x^{\prime}\right)\right)+(C, K)
$$


Remembering that $\ell \in L_{+}\left(E_{1}, E\right)$ and $h \in L_{x}(X, E)$ we have

$$
\begin{aligned}
y^{\prime}+\ell z^{\prime}-(y+\ell z) & \in v+\ell w+C \\
& \subset v+\ell w-h\left(\eta_{(x, y, z)}\left(x^{\prime}\right)\right)+h\left(\eta_{(x, y, z)}\left(x^{\prime}\right)\right)+C \\
& \subset(-C \backslash\{0\})^{c}+C+C \subset(-C \backslash\{0\})^{c},
\end{aligned}
$$

where $(v, w)$ is some element of $A_{(x, y, z)}\left(\eta_{(x, y, z)}\left(x^{\prime}\right)\right)$. Hence $y+\ell z \in H(s)$ and in fact $H_{2}(s)$ is a submap of $H(s)$. Finally, observe that the value of the objective of $\left(D_{2}\right)$ at a feasible solution $\ell$ such that $\ell(z)=0$ is $\{y\}$. Hence $\left(D_{2}\right)$ and $\left(C_{0}\right)$ have a common value and (DS $)$ is actually an exact dual of (CP $)$.

The two theorems above present a set-valued version of the results of $[2,3,4,5$, 6]. It should be worthwhile noticing that the existence of an operator $\ell$ solution of problem $\left(D_{1}\right)$ is guaranteed by the results of the previous section. If such an operator can be found, by taking $h=0$ we have $\ell$ and $h$ satisfying the condition of Theorem 6.3 .

Below we consider the case (b), when $E_{0}=\mathbb{R}, M=\mathbb{R}_{+}$, and we present a nonstandard dual for the problem

$$
\begin{aligned}
& \min F(x) \\
& G(x) \cap-K \neq \emptyset .
\end{aligned}
$$

For each feasible solution $(x, y, z)$ of $\left(\mathrm{CP}_{1}\right)$ we define the following set in the space $E \times E_{1}$ :

$$
N(x, y, z)=\left\{(0, z)+D(F, G)_{(x, y, z)} \eta_{(x, y, z)}(X)+(C, K)\right\} .
$$

Let us fix two vectors $e \in$ int $C, e_{1} \in$ int $K$ (this time we assume that int $K \neq \emptyset$ ). Define a function $s$ from $E \times E_{1}$ to $\mathbb{R} \cup\{+\infty\}$ by the rule: for every $(a, b) \in E \times E_{1}$,

$$
s(a, b)=\sup \left\{t \in \mathbb{R}:(a, b) \in(y, 0)+t\left(e, e_{1}\right)+N(x, y, z)\right\} .
$$

It is clear that $s$ is increasing in the first variable on its domain when the second one is zero. The order in $E$ is understood as generated by int $C \cup\{0\}$; in other words we are only dealing with weak minimality for $\left(\mathrm{CP}_{1}\right)$.

Denote by $S_{*}$ the set of such functions $s$ when $(x, y, z)$ runs over graph $(F, G)$ and $x$ runs over $X$. We then obtain a maximisation problem

$$
\begin{aligned}
& \max H(s) \\
& s \in S_{*}
\end{aligned}
$$

where $H(s)=\{d \in E: s(d, 0)=\min s((F, G)(X))\}$. 
THEOREM 6.4. Assume that $\left(C P_{1}\right)$ possesses a weak minimiser $(x, y, z)$, and that at this minimiser, the map $(F, G)$ is invex. Then $\left(D_{*}\right)$ is an exact dual for $\left(C P_{1}\right)$.

PrOOF: Observe that in the construction of duals by the axiomatic approach it is possible to allow $s$ to take the value $+\infty$. This does not change (WDA). Hence ( $D_{*}$ ) is actually a dual for $\left(\mathrm{CP}_{1}\right)$. To complete the proof we have to show that $\left(\mathrm{CP}_{1}\right)$ and $\left(D_{*}\right)$ have a common value. Let $s$ be defined by $(x, y, z)$ according to the formula (6.7). By invexity, for every $x^{\prime} \in X$,

$$
\begin{aligned}
(F, G)\left(x^{\prime}\right) & \subset(y, z)+D(F, G)_{(x, y, z)}\left(\eta_{(x, y, z)}\left(x^{\prime}\right)\right)+(C, K) \\
& \subset(y, 0)+N(x, y, z) .
\end{aligned}
$$

This implies that $s(a, b) \geqslant 0$ for each $(a, b) \in(F, G) X$. On the other hand, $s(y, 0) \leqslant$ 0 . Indeed if we suppose to the contrary that $s(y, 0)>0$, then there exist $t>0$ and $(v, w) \in D(F, G)_{(x, y, z)}\left(\eta_{(x, y, z)}(X)\right)$ such that $(0, z)+(v, w) \in-\operatorname{int}(C, K)$. Then for each vector $(\xi, \zeta) \in(C, K)^{\prime}$ we would have $\xi(v)+\zeta(w+z)<0$ contradicting Theorem 5.5. As $(y, 0) \in(F, G) x$ one gets finally $s(y, 0)=0$. Thus $s(a, b) \geqslant s(y, 0)=0$ for every $(a, b) \in(F, G) X$ which implies $y \in H(s)$, that is, it is a common value for both problems $\left(\mathrm{CP}_{1}\right)$ and $\left(\mathrm{D}_{*}\right)$.

In the rest of this section we discuss a possible application of the generalised Lagrangian duality approach which was recently developed in $[7,14]$ to the case of invex problems.

Let $Z$ be a parameter space which for convenience is assumed to be linear topological. Let $\mathcal{F}$ be a set-valued map from $X \times Z$ to $E$ such that $\mathcal{F}(x, 0)=F(x)$ for every $x \in X$. Problem (P) can be regarded as a particular case of the problem

$$
\begin{aligned}
& \min \mathcal{F}(x, z) \\
& x \in X_{\mathbf{0}},
\end{aligned}
$$

when $z$ takes the value zero.

Furthermore, let $\phi$ be a set of single-valued maps from $X \times Z$ to $E$ which are increasing in the first variable when the second one is zero. One can now define a Lagrangian map $L$ as a set-valued map from $X \times \phi$ to $E$ by the rule

$$
L(x, \varphi)=\bigcup_{z \in Z} \varphi(\mathcal{F}(x, z)+C, z)
$$

for every $x \in X, \varphi \in \phi$.

With this Lagrangian map one defines a dual map $H$ from $\phi$ to $E$ as

$$
H(\varphi)=\operatorname{Min} L\left(X_{0}, \varphi\right)
$$


and for a fixed subset $S$ of $\phi$ a maximisation problem:

$$
\begin{aligned}
& \max H(\varphi) . \\
& \varphi \in S
\end{aligned}
$$

If every element $\varphi \in S$ has the property that there is a single-valued map $\xi$ from $Z$ to $E$ such that $\xi(0)=0$ and $\varphi(x, z)=x+\xi(z)$, for all $x \in X, z \in Z$, then $\mathcal{F}\left(X_{0}, 0\right) \subset L\left(X_{0}, \varphi\right)$ and the weak duality relation holds. Hence (D) becomes a dual of (P). It is an exact dual if and only if there exists an optimal value $a_{0}$ of $(\mathrm{P})$ such that $a_{0} \in \operatorname{Min} \bigcup_{z \in Z}\left(\mathcal{F}\left(X_{0}, z\right)+C+\xi(z)\right)$. This inclusion means that $\xi$ is a subgradient of the map $z \rightarrow \mathcal{F}\left(X_{0}, z\right)+C$ at $\left(0, a_{0}\right)$. The reader is referred to [7, 14] for more details. For problem $\left(\mathrm{CP}_{0}\right)$ one can take $Z=E_{1}$ and

$$
\mathcal{F}(x, z)= \begin{cases}F(x) & \text { if } G(x) \cap(z-K) \neq \emptyset \\ \phi & \text { otherwise. }\end{cases}
$$

Problem $\left(\mathrm{P}_{z}\right)$ corresponding to this case is of the form:

$$
\begin{aligned}
& \min F(x) \\
& x \in X_{0}, G(x) \cap(z-K) \neq \emptyset .
\end{aligned}
$$

By choosing $\xi \in L_{+}\left(E_{1}, E\right)$ and $S$ as above, the dual map can be written as

$$
\begin{aligned}
H(\varphi) & =\operatorname{Min}\left\{F(x)+\xi(z): x \in X_{0}, G(x) \cap(z-K) \neq \emptyset\right\} \\
& =\operatorname{Min}\left\{(F+\xi G)\left(X_{0}\right)\right\} .
\end{aligned}
$$

It is exactly the map of formula (6.2), that we have obtained by the method of axiomatic duality.

\section{REFERENCES}

[1] J.P. Aubin and H. Frankowska, Set-valued analysis (Birkhaüser, 1990).

[2] A. Ben-Israel and B. Mond, 'What is invexity?', J. Austral. Math. Soc. (Series B) 28 (1986), 1-9.

[3] B.D. Craven, 'Invex functions and constrained local minima', Bull. Austral. Math. Soc. 25 (1981), 37-46.

[4] B.D. Craven, 'A modified Wolfe dual for weak vector minimization', Numer. Funct. Anal. Optim. 10 (1989), 899-907.

[5] B.D. Craven, 'Nonsmooth multiobjective programming', Numer. Funct. Anal. Optim. 10 (1989), 49-64. 
[6] B.D. Craven and B.M. Glover, 'Invex functions and duality', J. Austral. Math. Soc. (Series A) 39 (1985), 1-20.

[7] S. Dolecki and C. Malivert, 'General duality for vector optimization', (submitted).

[8] R.R. Egudo and M.A. Hanson, 'Multiobjective duality with invexity', J. Math. Anal. Appl. 126 (1987), 469-477.

[9] M.A. Hanson, 'On sufficiency of the Kuhn-Tucker conditions', J. Math. Anal. Appl. 80 (1981), 545-550.

[10] J. Jahn, Mathematical vector optimization in partially ordered linear spaces (Peter Lang, Frankfurt, 1986).

[11] D.T. Luc, Theory of vector optimization: Lecture Notes in Economics and Mathematical Systems 319 (Springer-Verlag, Berlin, Heidelberg, New York, 1989).

[12] D.T. Luc, 'Contingent derivatives of set-valued maps and applications to vector optimization', Math. Programming 50 (1991), 99-111.

[13] D.T. Luc and J. Jahn, 'Axiomatic approach to duality in optimization', (submitted).

[14] C. Malivert, Contributions à l'optimisation vectorielle, Thèse Université de Limoges, 1990.

[15] J.-P. Penot, 'Differentiability of relations and differential stability of perturbed optimization problems', SIAM J. Control Optim. 22 (1984), 529-551.

[16] Y. Tanaka, 'Note on generalized convex functions', J. Optim. Throry Appl. 66 (1990), 345-349.

[17] T. Weir and B. Mond, 'Generalized convexity and duality in multiple objective programming', Bull. Austral. Math. Soc. 39 (1989), 287-299.

\author{
Department of Mathematics \\ Faculty of Science \\ University of Limoges \\ Limoges, Cedex 87050 \\ France
}

